\title{
(อ) OPEN ACCESS \\ Drainage, irrigation and fibrinolytic therapy (DRIFT) for posthaemorrhagic ventricular dilatation: 10-year follow-up of a randomised controlled trial
}

\author{
Karen Luyt (D) , ${ }^{1,2}$ Sally L Jary, ${ }^{1}$ Charlotte L Lea, ${ }^{1}$ Grace J. Young ${ }^{3,4}$ David E Odd (D) , 1,5 \\ Helen E Miller, ${ }_{1}^{1}$ Grazyna Kmita, ${ }_{1}^{6}$ Cathy Williams, ${ }^{7,8}$ Peter S Blair, ${ }^{3,4}$ \\ William Hollingworth, ${ }^{3}$ Michelle Morgan, ${ }^{9}$ Adam P Smith-Collins, ${ }^{1,2}$ \\ Steven Walker-Cox, ${ }^{1}$ Kristian Aquilina, ${ }^{10}$ Ian Pople, ${ }^{11}$ Andrew G Whitelaw ${ }^{1}$
}

\begin{abstract}
- Additional material is published online only. To view please visit the journal online (http://dx.doi.org/10.1136/ archdischild-2019-318231).

For numbered affiliations see end of article.
\end{abstract} Dr Karen Luyt, Neonatal Neurology, Bristol Medical School, University of Bristol Faculty of Health Sciences, Bristol BS8 1QU, UK; karen.luyt@bristol.ac.uk

Received 12 September 2019 Revised 21 January 2020 Accepted 27 January 2020 Published Online First 4 July 2020

\section{Correspondence to}

\section{ABSTRACT \\ Background Progressive ventricular dilatation after intraventricular haemorrhage (IVH) in preterm infants has a very high risk of severe disability and death. Drainage, irrigation and fibrinolytic therapy (DRIFT), in a randomised controlled trial $(R C T)$, reduced severe cognitive impairment at 2 years.}

Objective To assess if the cognitive advantage of DRIFT seen at 2 years persisted until school age.

Participants The RCT conducted in four centres recruited 77 preterm infants with IVH and progressive ventricular enlargement over specified measurements. Follow-up was at 10 years of age.

Intervention Intraventricular injection of a fibrinolytic followed by continuous lavage, until the drainage was clear, and standard care consisting of control of expansion by lumbar punctures and if expansion persisted via a ventricular access device.

Primary outcome Cognitive quotient (CQ), derived from the British Ability Scales and Bayley III Scales, and survival without severe cognitive disability.

Results Of the 77 children randomised, 12 died, 2 could not be traced, 10 did not respond and 1 declined at 10-year follow-up. 28 in the DRIFT group and 24 in the standard treatment group were assessed by examiners blinded to the intervention. The mean CQ score was $69.3(S D=30.1)$ in the DRIFT group and 53.7 $(S D=35.7)$ in the standard treatment group (unadjusted $p=0.1$; adjusted $p=0.01$, after adjustment for the prespecified variables sex, birth weight and IVH grade). Survival without severe cognitive disability was $66 \%$ in the DRIFT group and 35\% in the standard treatment group (unadjusted $p=0.019$; adjusted $p=0.003$ ).

Conclusion DRIFT is the first intervention for posthaemorrhagic ventricular dilatation to objectively demonstrate sustained cognitive improvement. Trial registration number ISRCTN80286058.

\section{INTRODUCTION}

(c) Author(s) (or their employer(s)) 2020. Re-use permitted under CC BY. Published by BMJ.

To cite: Luyt K, Jary SL, Lea $\mathrm{CL}$, et al. Arch Dis Child Fetal Neonatal Ed

2020:105:F466-F473.
Intraventricular haemorrhage (IVH) remains one of the most serious complications of preterm birth. It is the most common cause of brain injury in preterm infants ${ }^{1}$ and the primary risk factor for special educational needs at school age. ${ }^{2}$ Large IVHs cause a progressive obliterative arachnoiditis, disturbing the flow and absorption of cerebrospinal fluid (CSF), ${ }^{3}$ thereby causing posthaemorrhagic

\section{What is already known on this topic?}

- Progressive ventricular dilatation after intraventricular haemorrhage (IVH) in preterm infants has a very high risk of severe disability and death.

- Several interventions tested in randomised controlled trials (RCTs) have failed to reduce neurodisability in preterm infants with IVH and posthaemorrhagic ventricular dilatation (PHVD).

- Drainage, irrigation and fibrinolytic therapy (DRIFT), in an RCT, reduced severe cognitive disability at 2 years of age.

\section{What this study adds?}

- DRIFT achieved a sustained reduction of severe cognitive disability at school age, the first intervention for IVH with PHVD to objectively demonstrate long-term benefit.

- The findings are applicable to preterm infants with PHVD in well-resourced healthcare settings.

- The proof of principle that secondary brain injury is reduced by washing away the harmful debris of IVH in a controlled way has been established.

ventricular dilatation (PHVD). Around 70\% of very low birthweight (VLBW) infants with severegrade IVH develop persistent PHVD, and a third of infants with PHVD require surgical drainage of CSF by permanent ventriculoperitoneal (VP) shunt to control ventricular expansion. ${ }^{4}$ The raised pressure, distortion, and neurotoxic and inflammatory effects of blood in the ventricular system cause progressive brain injury and subsequent neurodisability, which is often severe. ${ }^{5}$ Infants under $1000 \mathrm{~g}$ who went on to shunt surgery had very high rates of motor, cognitive and multiple disabilities. ${ }^{6}$

A European survey found the most common approach in VLBW infants with PHVD was repeated lumbar punctures (LPs) followed by insertion of a ventricular access device (VAD) to enable regular tapping of CSF and control of ventricular expansion. If the need for tapping persists, a VP 
shunt is then inserted weeks later when the blood and protein have cleared from the CSF and the infant's weight has reached $2 \mathrm{~kg} .{ }^{7} \mathrm{VAD}$ and VP shunt insertion are associated with significant risk of infection and malfunction. ${ }^{8}$

Several interventions tested in randomised controlled trials (RCTs) have failed to reduce neurodisability rates as a result of PHVD. ${ }^{9}$ Drainage, irrigation and fibrinolytic therapy (DRIFT) $^{10-12}$ was developed due to the unsatisfactory results of other treatments. The objective is to remove proinflammatory cytokines, free iron and old blood from within the ventricles, and reduce both pressure and distortion.

The DRIFT RCT was conducted between 2003 and 2006. ${ }^{10}$ There were no differences in the need for VP shunt or death at 6 months. However, at 2 years post-term, severe disability or death was significantly reduced in the DRIFT group. ${ }^{11}$

We have followed up these patients at 10 years of age to determine if the cognitive advantage seen with DRIFT at 2 years continued through to school age. The secondary objectives were to assess long-term visual, sensorimotor function and emotional/ behavioural difficulties. We hypothesised that DRIFT would reduce severe cognitive disability at school age.

\section{METHODS}

\section{Initial study}

DRIFT is a surgical approach developed in Bristol. Temporary frontal and left occipital ventricular catheters are inserted under anaesthesia. Tissue plasminogen activator is injected intraventricularly at a subsystemic dose. The ventricles are irrigated by artificial CSF through a frontal catheter. When the ventricular system has been cleared of blood and debris, catheters are removed.

After feasibility testing showed DRIFT was technically possible and promising, ${ }^{12}$ the DRIFT RCT started recruiting in $2003 .{ }^{10}$

Eligible babies were preterm, had had IVH and had expanded cerebral ventricles over predetermined measurements. In total 77 babies (54 in Bristol (UK), 20 in Katowice (Poland), 2 in Glasgow (UK) and 1 in Bergen (Norway)) were randomised during 20032006 to either DRIFT or standard treatment, which consisted of LPs to control excessive expansion and pressure symptoms. If repeated LPs were needed, a VAD was surgically inserted to facilitate tapping of CSF. Every infant randomised to DRIFT received DRIFT, and no infant in the standard treatment group received DRIFT. At the time of intervention it was not possible to blind clinicians; however, all subsequent researchers were unaware of treatment group allocation. Full details of the trial have been published. ${ }^{10}$

Of the 77 babies randomised, 69 survived until 2 years. Severe cognitive disability (Bayley Mental Development Index (MDI) 3 SD below the mean) was 31\% in the DRIFT group and 59\% in the standard treatment group (adjusted OR: 0.17 (95\% CI 0.05 to 0.57$)$ ), and the difference in median MDI score was more than 18 points. ${ }^{11}$

\section{Follow-up study}

The follow-up study was designed with input from children and parents who had taken part in the initial feasibility study. Assessments consisted of cognitive, motor and visual ability, presence and severity of cerebral palsy (CP), and parental completion of a vision and behavioural inventory. Children in Poland did not have visual or motor assessments. All assessments were performed between February 2015 and April 2016. All outcome assessors were blinded to treatment allocation.

The primary hypothesis was whether DRIFT would reduce severe cognitive disability in children assessed at school age.
Imbalances in covariates affecting cognition were seen at randomisation and in the previous 2-year follow-up. Consequently, for this work we prespecified in the statistical analysis plan adjustment for birth weight, IVH grade and sex, with approval by the independent National Institute for Health Research follow-up study steering committee (including an independent statistician). The secondary outcomes were also adjusted for age, as the follow-up ages ranged from 8 to 12 years. Primary, secondary and exploratory analyses described here were prespecified in the study protocol (https://www.journalslibrary.nihr.ac.uk/programmes/hta/123561/\#/).

The follow-up study was funded by the National Institute of Health Research (Health Technology Assessment), for which a report/monograph has been published in full. ${ }^{13}$

\section{Primary outcome}

\section{Cognitive disability at school age}

Cognitive assessments were undertaken by two child psychologists. The British Ability Scales-III was used for children with a developmental age of $3+$ years. ${ }^{14}$ For children who did not meet this threshold, the Bayley Scales of Infant and Toddler Development-III was administered. ${ }^{15}$ The final scores were in the format of a cognitive quotient (CQ; from 0 to $100+$ ), based on the division of their developmental age equivalent by their actual age (multiplied by 100). ${ }^{16}$ The primary analysis was based on the cognitive scores of surviving children, although a sensitivity analysis was performed including children who died (due to disability), where the CQ for these children could reasonably be assumed to be 0 . Several sensitivity analyses were carried out, including the use of a binary outcome (death or severe cognitive disability), where all deaths over the 10-year period and deaths post 2 years that were deemed related to disability were included as negative outcomes. For patients who died after the 2-year follow-up, where the cause of death was unknown, we assumed that their death was due to disability if they had severe disability at 2 years. Severe cognitive disability was defined as a cognitive score below $3 \mathrm{SD}$ of the population mean.

\section{Secondary outcomes}

Cerebral visual function

For the main visual outcomes, parents were asked about their child's vision, describing them as 'No concerns', 'Normal with Correction', 'Useful but not fully correctable' and 'Blind or perceives light only'. A binary outcome was created that split these into good visual outcome (no concerns/normal with correction) and poor visual outcome (useful but not fully correctable/ blind or perceives light only). Parents were asked 23 questions about their child's visual behaviour from an inventory identifying examples of vision processing impairment. ${ }^{17} \mathrm{~A}$ mean score was created.

\section{Sensorimotor disability}

Children were assessed using the Movement Assessment Battery for Children-2 (MABC-2) by a paediatric physiotherapist. ${ }^{18}$ The presence and severity of CP were also compared between the two groups according to the recommendations of the Surveillance of Cerebral Palsy in Europe, using the Gross Motor Function Classification System. ${ }^{19}$ All children with $\mathrm{CP}$ were classified as having severe sensorimotor disability, moderate if MABC- 2 scores were between 57 and 67, and none if $\mathrm{MABC}-2>67$. 


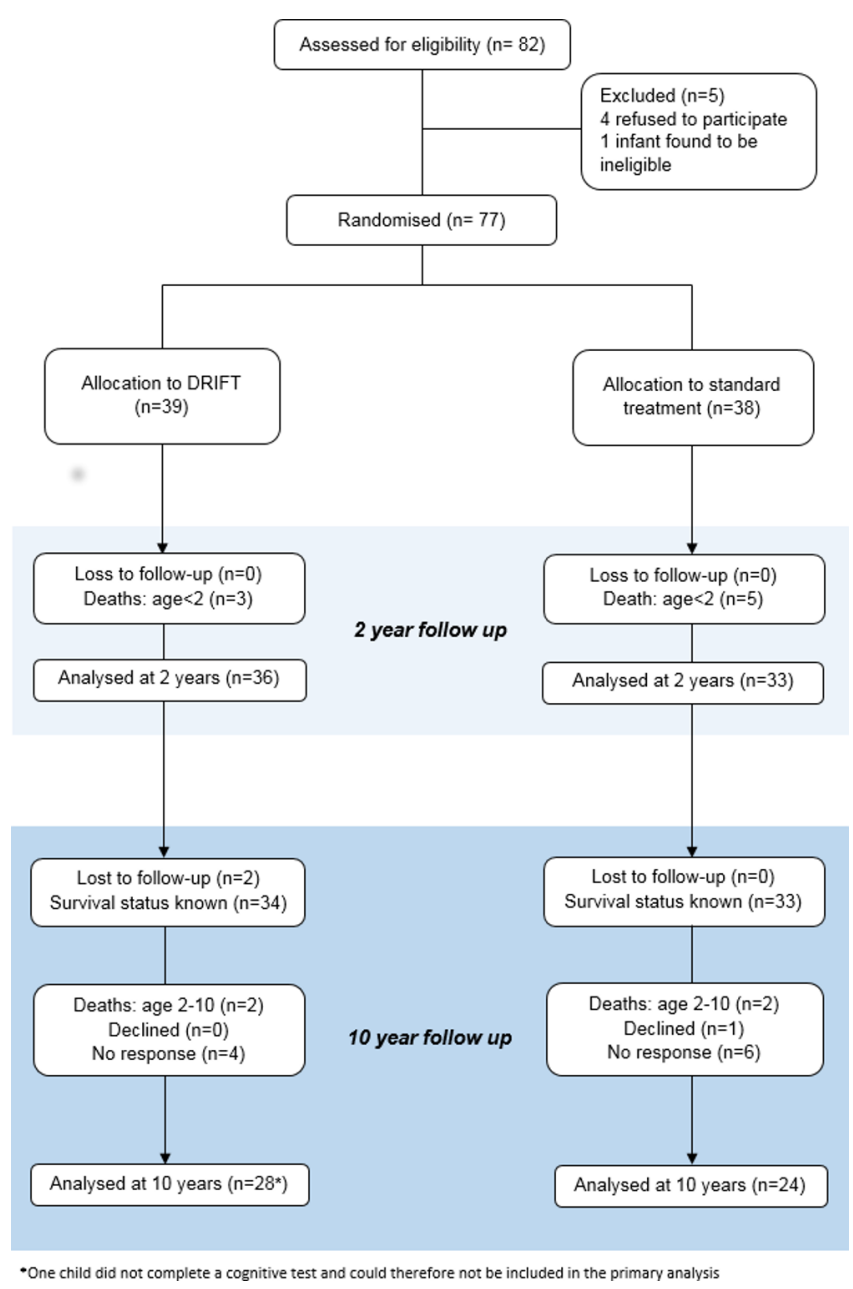

Figure 1 Drainage, irrigation and fibrinolytic therapy (DRIFT) participant flow.

\section{Emotional/behavioural function}

Parents were asked to complete the Strengths and Difficulties Questionnaire (SDQ), which assesses child behaviour. The final score classifies the child as having normal or abnormal behaviour. ${ }^{20}$

\section{Power calculation}

Based on the effect size of DRIFT treatment on severe cognitive disability at 2 years, ${ }^{11}$ a two-group continuity-corrected $\chi^{2}$ test with $5 \%$ two-sided significance level would have $80 \%$ power to detect the difference in severe cognitive disability between a standard treatment group proportion of 59\% and OR of 0.17 (ie, an intervention proportion of $19.7 \%$ ) when the sample size in each group is 28 . Assuming $90 \%$ follow-up rate, 60 infants (30 in each group) would have $97 \%$ power (with an alpha of $5 \%$ ) to detect a mean cognitive score difference of 1 SD (commonly 15 points) between the DRIFT and the standard treatment group.

\section{Statistical analysis}

All statistical analyses were performed using STATA V.14.1 software. All $\mathrm{p}$ values were two-sided and considered significant if $\mathrm{p}<0.05$.

\section{RESULTS}

\section{Study population and participant flow}

Figure 1 shows the layout of the trial and the different levels of dropout and analyses. Seventy-seven babies were originally recruited to either receive DRIFT (39 infants) or standard treatment (38 infants).

At 2-year follow-up there had been eight deaths due to complications of prematurity, but no loss to follow-up. Approximately 8 years later (between September 2015 and April 2016), parents were contacted and asked to take part in the 10-year follow-up study. Two patients (both in the DRIFT group) could not be traced. This left 67 patients where the survival status was known. Of these, there were 2 deaths in the DRIFT group, 2 deaths in the standard treatment group (all 4 were associated with severe disability), 1 patient who declined to take part in the follow-up (standard treatment group) and 10 who gave no response (4 DRIFT, 6 standard), leaving 52 available for assessment (28 DRIFT, 24 standard). For the primary outcome we obtained 51 children's CQ score: 27 in the DRIFT group and 24 in the standard treatment group.

\section{Baseline data}

For the 52 children available for follow-up assessments at 10 years, there were imbalances seen in sex and birth weight (table 1). In the DRIFT group, 22 (79\%) children were male, whereas in the standard treatment group $63 \%$ were male. The mean birth weight was $1322 \mathrm{~g}$ in the standard treatment group and $1102 \mathrm{~g}$ in the DRIFT group.

\section{Primary analyses}

Primary outcomes

CQ scores were relatively normally distributed with an overall mean of 62.0 and a median of 68.7. Figure 2 shows the distribution of scores by treatment allocation. Children receiving DRIFT had a mean quotient score of 69.3 (median 72.3) vs 53.7 (median 44.6) for children receiving standard treatment. The maximum CQ score was 130.6 (DRIFT group), indicating a cognitive ability that is $30 \%$ higher than we would expect to see at their age (developmental age 30\% higher than actual age). The highest CQ score achieved in the standard treatment group was 107.2. Only two children had CQ score below 30 (profound cognitive disability) in the DRIFT group, compared with seven children in the standard treatment group.

Table 2 shows the results of the primary analysis, including and excluding deaths. Given the larger than expected attrition/death rate, precision was lower than predicted, exacerbated further by the large SD for the CQ. Despite this, the results are in parallel with those at 2 years, with crude estimates giving weak evidence that the DRIFT intervention increases cognitive ability at 10 years $(p=0.096)$. After adjusting for the prespecified variables sex, birth weight and IVH grade, this evidence was strengthened and indicated that children in the DRIFT group had, on average, CQ scores 23.47 points higher than those who received standard treatment $(\mathrm{p}=0.009)$. This translates into a developmental cognitive advantage of 2.5 years (95\% CI 0.5 to 4.4 ).

\section{Sensitivity analyses}

The binary outcome used in the 2-year follow-up was replicated at 10 years, resulting in 21 of $32(66 \%)$ patients surviving without severe cognitive disability in the DRIFT group compared with 11 of 31 (35\%) patients in the standard treatment group (adjusted OR 7.69 (1.96 to 30.11), p=0.003). Survival without severe cognitive disability (removing deaths before 2 years) also gave very similar results to the continuous CQ outcome (unadjusted OR 3.58 (1.16 to 11.04 ), $\mathrm{p}=0.026$; adjusted OR 9.96 (2.12 to 46.67$), \mathrm{p}=0.004)$. The number needed to treat $(\mathrm{NNT})$ to prevent one death or case of severe cognitive disability was 
Table 1 Characteristics of participants assessed at 10 years by trial allocation

\begin{tabular}{|c|c|c|c|c|c|c|}
\hline & & DRIFT & & & Standard & \\
\hline & $\mathbf{n}$ & Mean (SD) or $n(\%)$ & & $\mathbf{n}$ & Mean (SD) or $n(\%)$ & \\
\hline Total number of participants & 28 & & & 24 & & \\
\hline Centre & & & & & & \\
\hline Bristol, UK & 28 & {[} & $23(82 \%)$ & 24 & {[} & $19(79 \%)$ \\
\hline Katowice, Poland & & & $3(11 \%)$ & & & $4(17 \%)$ \\
\hline Glasgow, UK & & & $1(4 \%)$ & & & $1(4 \%)$ \\
\hline Bergen, Norway & & L & $1(4 \%)$ & & L & $0(0 \%)$ \\
\hline Sociodemographics at birth & & & & & & \\
\hline Age at randomisation (days) & 28 & & $18.68(5.00)$ & 24 & & $19.17(4.53)$ \\
\hline Sex: male* & 28 & & $22(79 \%)$ & 24 & & $15(63 \%)$ \\
\hline Clinical characteristics at birt & & & & & & \\
\hline Birth weight $(\mathrm{g})^{*}$ & 28 & & $1101.89(335.54)$ & 24 & & $1322.46(534.68)$ \\
\hline Gestation (weeks) & 28 & & $27.64(2.56)$ & 24 & & $28.50(3.05)$ \\
\hline Grade of IVH: 4 & 28 & & $14(50 \%)$ & 24 & & $11(46 \%)$ \\
\hline Maternal age at birth & 14 & & $28.50(6.99)$ & 12 & & $28.17(6.32)$ \\
\hline $\begin{array}{l}\text { Median IMD 2010, postcode } \\
\text { at birtht (IQR) }\end{array}$ & 18 & & $26.7(8.2-36.5)$ & 18 & & $27.6(11.2-45.8)$ \\
\hline Measures at 2 years & & & & & & \\
\hline Experienced second IVH* & 28 & & $8(29 \%)$ & 24 & & $3(13 \%)$ \\
\hline VP shunt & 28 & & $11(39 \%)$ & 24 & & $8(33 \%)$ \\
\hline Ventricular access device* & 28 & & $13(46 \%)$ & 24 & & $19(79 \%)$ \\
\hline Measures at 10 years & & & & & & \\
\hline $\begin{array}{l}\text { Age at } 10 \text {-year assessment } \\
\text { (years) }\end{array}$ & 28 & & $10.56(1.07)$ & 24 & & $10.76(1.06)$ \\
\hline Weight (kg) & 28 & & $35.41(10.05)$ & 23 & & $34.73(10.51)$ \\
\hline Height $(\mathrm{cm})$ & 28 & & $139.09(12.22)$ & 23 & & $142.26(11.34)$ \\
\hline Head circumference $(\mathrm{cm})$ & 28 & & $52.88(2.53)$ & 23 & & $52.00(3.43)$ \\
\hline Maternal education* & & & & & & \\
\hline Left school at 16 & 28 & {[} & $10(36 \%)$ & 23 & {[} & $11(48 \%)$ \\
\hline Further education & & & $6(21 \%)$ & & & $5(22 \%)$ \\
\hline University degree & & L & $12(43 \%)$ & & 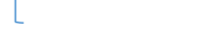 & $7(30 \%)$ \\
\hline
\end{tabular}

${ }^{*}$ Difference of $10 \% / 0.5$ SD or higher between the groups.

tEnglish Index of Multiple Deprivation (IMD) 2010 scores, UK Data Service Census Support (http://geoconvert.mimas.ac.uk/). Higher scores indicate higher levels of deprivation. IMD based on the children's home postcode at birth for those residing in England only.

DRIFT, drainage, irrigation and fibrinolytic therapy; IVH, intraventricular haemorrhage; VP, ventriculoperitoneal.

3. More details on cause of death and sensitivity analyses are included in the online supplementary appendix.

\section{Secondary outcomes}

Parent-reported vision

A higher proportion of children from the DRIFT group had 'good visual outcome', but there was high variance and this was not statistically significant (adjusted OR 3.73 (95\% CI 0.66 to 21.14), $\mathrm{p}=0.136)$. A small difference was found in the question inventory mean score (adjusted mean difference - 0.12 (95\% CI -0.47 to 0.24$), \mathrm{p}=0.502$ ) (table 3 ).

\section{Sensorimotor disability}

There was no difference in sensorimotor disability, with severe, moderate and no sensorimotor disability percentages of $85 \%$, $7 \%$ and $7 \%$ for patients in the DRIFT group, respectively, compared with $76 \%, 10 \%$ and $14 \%$ in the standard treatment group (adjusted OR 3.66 (0.33 to 40.34 ), $\mathrm{p}=0.290)$. There was no significant difference in the percentages with CP or severity of CP between the two groups (61\% vs 58\% for DRIFT and standard treatment, respectively) (table 3).
Emotional/behavioural function

For the SDQ total score, higher values indicated more 'abnormal' behaviour. There was no difference between the two groups (adjusted mean difference 2.01 (95\% CI -2.78 to 6.81), $\mathrm{p}=0.401)$.

\section{Sensitivity/subgroup analyses}

Additional sensitivity analyses, described in the online supplementary appendix, were carried out that include multiple imputation and adjustment for centre, all of which were consistent with the primary outcome (online supplementary eTable 1). We did not find any subgroup effects to suggest that DRIFT was more effective in certain subgroups, although it needs to be emphasised that we were not powered to detect any differences between subgroups (online supplementary eTable 2). Maternal educational attainment was imbalanced at 10 years, with a greater proportion of mothers in the DRIFT group accessing university education (table 1 ). In a post-hoc sensitivity analysis, we adjusted for maternal level of education (online supplementary eTable 1). Adjustment for maternal education (measured 10 years after randomisation) resulted in a slight attenuation of 


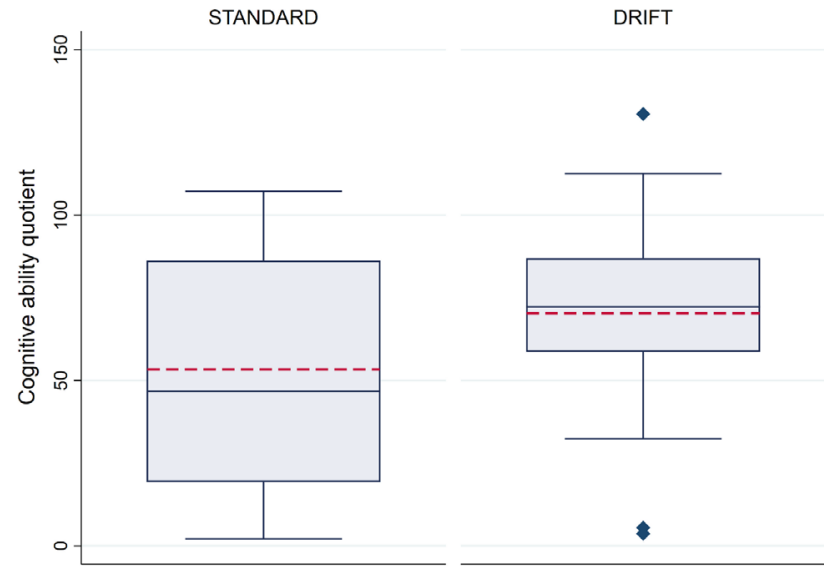

Figure 2 Cognitive quotient, by trial allocation. The box plot indicates the median (central blue line in the box), mean (red dashed line), 25th percentile (bottom line of the box), 75th percentile (top line of the box), and the whiskers for the minimum and maximum values (excluding outliers). The diamonds beyond these whiskers are the outliers, which are observations that lie at an abnormal distance from other values. Outliers are defined as those higher than $1.5 \times 1 \mathrm{QR}+75$ th percentile or lower than 25th percentile-1.5× IQR. DRIFT, drainage, irrigation and fibrinolytic therapy.

effect size with an 11.5 point CQ advantage (unadjusted $\mathrm{p}=0.2$ ) and a significant 20.1 point CQ advantage after adjustment for the prespecified variables sex, birth weight and IVH grade (adjusted $\mathrm{p}=0.02$ ) with DRIFT.

Additional data from parental history were available regarding special education needs in children. After adjustment, those in the DRIFT group showed a trend towards lower odds of special school attendance in the preceding 12 months compared with those in the standard treatment group (OR 0.27 (95\% CI 0.07 to 1.05$), p=0.059)$. This suggests that there may be a benefit in terms of educational attainment from DRIFT treatment.

\section{DISCUSSION}

\section{Summary of findings}

DRIFT treatment of preterm infants with severe IVH and PHVD improves cognitive ability at 10 -year follow-up when taking into account birth weight, IVH grade and sex. There were no significant differences in the secondary outcomes: parent-reported visual impairment, sensorimotor disability or emotional/behavioural difficulties. Surrogate markers of functional ability (special education) showed a trend towards long-term benefits of DRIFT treatment.

Crucially, infants who received DRIFT were almost twice as likely to survive without severe cognitive disability than those who received standard treatment. While the CIs were wide, the point estimate suggests that the NNT for DRIFT to prevent one death or one case of severe cognitive disability was 3 .

\section{Strengths and limitations}

The strength of this study is the long-term follow-up to middleschool age, which strengthens the validity of conclusions around cognitive ability. In neonatal interventional trials, long-term follow-up is challenging as families move. It requires active buy-in from both children and their parents and a significant time commitment for families. Where a significant proportion of survivors have severe neurodisabilities, as seen with PHVD, the logistics to return for follow-up assessment become even more challenging.

Where children have a very wide range of abilities, precise cognitive scoring becomes a significant challenge. Our approach to cognitive assessment achieved CQ in children of all abilities. Inclusion of special education as a pragmatic post-hoc outcome gives some idea of the likely gains going forward into an independent adulthood.

Ongoing family involvement and the organisation of the British National Health Service ensured a very high follow-up rate at school age in the UK. Only two patients had an unknown survival status at 10 years, and best and worst case scenarios were also explored (online supplementary eTable 1).

While the higher CQ score results were apparent in all analyses, the effect did not reach conventional levels of statistical significance in the unadjusted analyses of mean CQ scores. However, important characteristics were unbalanced at randomisation and at 10-year follow-up, with a larger proportion of higher risk cases in the DRIFT group. In the DRIFT group, infants were significantly smaller, less mature, with greater proportion of male and had more severe-grade IVH, and consequently adjustments for the imbalances were prespecified in the analysis plan. Of note, the association seen in the binary outcome (survival without severe cognitive disability) was clear in the unadjusted and adjusted analyses. Another limitation was that maternal education was not collected at birth, and our use of a proxy measure of maternal education at 10 years is difficult to interpret, especially if educational attainment was gained after baseline. We have adjusted for maternal educational level, which may be a useful proxy but may not give a full picture of family functioning. Details about use of rehabilitative services

\begin{tabular}{|c|c|c|c|c|c|}
\hline Variable & $\begin{array}{l}\mathrm{n} \\
\text { (DRIFT:standard) }\end{array}$ & $\begin{array}{l}\text { DRIFT } \\
\text { Mean (SD) or } n(\%)\end{array}$ & $\begin{array}{l}\text { Standard } \\
\text { Mean (SD) or } n(\%)\end{array}$ & $\begin{array}{l}\text { Crude difference }(95 \% \mathrm{Cl}), \mathrm{p} \\
\text { value }\end{array}$ & $\begin{array}{l}\text { Adjusted difference }(95 \% \mathrm{Cl}) \text {, } \\
\text { p value* }\end{array}$ \\
\hline \multicolumn{6}{|l|}{ Primary outcome } \\
\hline Cognitive ability quotient & $27: 24$ & $69.33(30.06)$ & $53.68(35.70)$ & $15.65(-2.86$ to 34.16$), 0.096 \dagger$ & 23.47 (6.23 to 40.71$), 0.009 \dagger$ \\
\hline \multicolumn{6}{|l|}{ Sensitivity analyses } \\
\hline Cognitive ability quotientł & $29: 26$ & $64.55(34.04)$ & $49.55(37.22)$ & $15.00(-4.28$ to 34.27$), 0.125 \dagger$ & 22.33 (4.77 to 39.89 ), $0.014 \dagger$ \\
\hline Alive and without severe cognitive disability§ & $29: 26$ & $21(72 \%)$ & $11(42 \%)$ & 3.58 (1.16 to 11.04$), 0.026$ १ & 9.96 (2.12 to 46.67$), 0.004 \uparrow$ \\
\hline Alive and without severe cognitive disability* * & $32: 31$ & $21(66 \%)$ & $11(35 \%)$ & 3.47 (1.23 to 9.78$), 0.019$ ๆ & 7.69 (1.96 to 30.11$), 0.0039$ \\
\hline
\end{tabular}

*Adjusted for sex, birth weight and grade of IVH.

thinear regression (difference in means).

¥Giving children who have died post 2 years a score of 0 .

§Including all 4 deaths after 2 years as a negative outcome.

१logistic regression (OR).

** Including all 12 deaths as a negative outcome (akin to the study results at 2 years).

DRIFT, drainage, irrigation and fibrinolytic therapy; IVH, intraventricular haemorrhage. 
Original research

Table 3 Secondary outcomes

\begin{tabular}{|c|c|c|c|c|c|}
\hline & $\begin{array}{l}\mathrm{n} \\
\text { (DRIFT:standard) }\end{array}$ & $\begin{array}{l}\text { DRIFT } \\
\mathrm{n}(\%) \text { or mean (SD) }\end{array}$ & $\begin{array}{l}\text { Standard } \\
\mathrm{n}(\%) \text { or mean (SD) }\end{array}$ & $\begin{array}{l}\text { Unadjusted difference } \\
(95 \% \mathrm{Cl}), \mathrm{p} \text { value }\end{array}$ & $\begin{array}{l}\text { Adjusted difference* } \\
(95 \% \mathrm{Cl}), \mathrm{p} \text { value }\end{array}$ \\
\hline \multicolumn{6}{|l|}{ Parent-reported visual function } \\
\hline Good vision & $27: 24$ & $23(85 \%)$ & $17(71 \%)$ & $2.37(0.60$ to 9.40$), 0.22 \dagger$ & 3.73 (0.66 to 21.14$), 0.14 \dagger$ \\
\hline \multicolumn{6}{|l|}{ Sensorimotor disability } \\
\hline None & & $2(7 \%)$ & $3(14 \%)$ & & \\
\hline \multicolumn{6}{|l|}{ Cerebral palsy } \\
\hline Diagnosed with cerebral palsy (\%) & $28: 24$ & $17(61 \%)$ & $14(58 \%)$ & 1.10 (0.36 to 3.35$), 0.86 t$ & $0.37(0.07$ to 2.00$), 0.25 \dagger$ \\
\hline GMFCS level 1 & & $7(41 \%)$ & $5(36 \%)$ & & \\
\hline GMFCS level 2 & & $4(24 \%)$ & $3(21 \%)$ & & \\
\hline GMFCS level 3 & & $2(12 \%)$ & $0(0 \%)$ & & \\
\hline Total score & $28: 22$ & $14.89(8.48)$ & $13.36(6.59)$ & 1.53 (-2.89 to 5.94$), 0.49 \ddagger$ & $2.01(-2.78$ to 6.81$), 0.40 \ddagger$ \\
\hline
\end{tabular}

${ }^{*}$ Adjusted for age, sex, birth weight and grade of IVH.

tLogistic regression (OR).

‡Linear regression (difference in means).

$\S 0$ dinal logistic regression (OR per increase in category).

ๆChildren with cerebral palsy were categorised as ambulant (GMFCS level 1-2) or non-ambulant (GMFCS level 3-5).

DRIFT, drainage, irrigation and fibrinolytic therapy; GMFCS, Gross Motor Function Classification System; IVH, intraventricular haemorrhage.

were not collected as these data are not formally available in the UK and it was not reasonable to expect families to reliably recall the type and level of intervention over a period of 9-10 years after discharge from the neonatal unit.

The main limitation is reduced precision of results due to the size of the trial. This intervention was innovative and invasive, and for safety reasons the trial had stringent stopping criteria which limited the achieved sample size. This unfortunately resulted in a lower sample size than was required to give $80 \%$ power for the primary outcome (CQ), and consequently wide CIs around the point estimates for both the continuous and binary outcomes.

\section{Interpretation of results}

Preterm infants with severe IVH and PHVD have a very high reported rate of neurodisability. The National Institute of Child Health and Development study, the largest of its kind, studied preterm infants with severe IVH (grade 3 and 4), of whom almost 25\% had PHVD. ${ }^{6}$ At 18-22 months corrected age, 68\% of children with severe-grade IVH and PHVD were reported to have moderate cognitive impairment (MDI below 2 SD) and 41\% had severe cognitive impairment (MDI below 3 SD). The presence of haemorrhagic parenchymal infarction, in addition to PHVD, increased the risk of CP to between $80 \%$ and $90 \%$.

In our study follow-up at 10 years found similar severe cognitive disability rates: $52 \%$ in the standard treatment arm. The proportion of children with severe cognitive disability with DRIFT was $21 \%$. This paper further supports that the DRIFT process improves outcome in this group of infants, with evidence of improvement in the number of infants with a very low cognitive score and an overall increase in the mean cognitive score. With the precision available in this trial, we were unable to identify if DRIFT has a disproportionate effect across this range. Children who received DRIFT were also more likely to attend mainstream schools. The reduction in severe cognitive disability seen with this intervention is likely to translate into the ability to lead more independent lives into adulthood.

DRIFT had an effect on cognition but did not appear to improve motor function. Of the children, 50\% (DRIFT) and 46\% (standard care) had haemorrhagic parenchymal infarctions (grade $4 \mathrm{IVH})$. The most likely explanation for the disproportionate effect on cognition is that simple irrigation, although effective at reducing secondary global neurotoxicity and damage to cortical and subcortical tissue, is not sufficient to promote tissue regeneration in critical motor tracts after significant parenchymal infarction. Motor outcome is likely primarily determined by the initial amount of acute tissue injury/loss incurred by a large focal haemorrhagic parenchymal infarction. There was a strong trend towards improved visual function, but this did not reach statistical significance due to marked variability of outcome.

\section{Implications for practice in the context of current standard treatments and other research}

DRIFT is the first and only intervention for PHVD in preterm infants to demonstrate long-term benefit in an RCT. The proof of principle that secondary brain injury is reduced by washing away the harmful debris of IVH in a controlled way with DRIFT has been established. This is a hugely promising development in a group of infants with a high rate of long-term neurodisability.

Existing standard treatment with relief of intracranial pressure by CSF drainage through LP or insertion of a VAD results in worse outcomes than those seen with DRIFT. The recently published data from the Early vs Late Ventricular Intervention Study (ISRCTN43171322), a multicentre RCT comparing two treatment thresholds for VAD insertion after PHVD, have not revealed benefits from commencing standard treatment at a lower threshold. The short-term outcomes show no benefit from the lower threshold and significantly more invasive procedures. ${ }^{21}$ 
There was no significant difference in mortality or neurodevelopmental outcome at 2 years of age. ${ }^{22}$

Alternative surgical interventions are being tested. A feasibility study of ventricular endoscopic lavage demonstrated fewer short-term complications and need for VP shunts in comparison with standard treatment in historical controls. However, there is a lack of long-term data on outcomes from these children. ${ }^{23}$

DRIFT then offers the only proven option for improving outcomes after PHVD in preterm infants. The aim of DRIFT is to improve neurodevelopmental outcomes, and therefore while there appears to be a net benefit to the infant this would need to be balanced with the potential risks (eg, infection, secondary haemorrhage and rapid fluctuation of intracranial pressure). The expertise required for skilled insertion of two ventricular catheters into a tiny infant, followed by prolonged accurate monitoring of intracranial pressure, adjustment of drainage rate and detection of catheter blockage, all done with immaculate aseptic technique, is significant. The complexity, risks and rarity of candidates are roughly comparable with those of extracorporeal membrane oxygenation, and implementation of DRIFT as a clinical service would likely require a small number of highly specialised centres in order to achieve adequate throughput of patients to develop and maintain such expertise, for example, four or five for the UK. For comparison, Haukeland Hospital in Bergen is the only centre in Norway (population 5.3 million) providing DRIFT service to referred infants.

\section{CONCLUSIONS}

PHVD is associated with high levels of severe neurodisability with marked detrimental effect on cognitive function. DRIFT is the first intervention to objectively demonstrate sustained cognitive improvement in preterm infants with PHVD that is sustained into middle-school age. Although invasive and technically demanding, this treatment approach provides clear improvements in outcome and deserves detailed further evaluation.

\footnotetext{
Author affiliations

${ }^{1}$ Neonatal Neurology, Bristol Medical School, Faculty of Health Sciences, University of Bristol, Bristol, UK

${ }^{2}$ Neonatal Intensive Care Unit, St Michael's Hospital, University Hospitals Bristol NHS Foundation Trust, Bristol, UK

${ }^{3}$ Population Health Sciences, Bristol Medical School, University of Bristol, Bristol, UK ${ }^{4}$ Bristol Randomised Trials Collaboration (BRTC), Bristol Trials Centre, Bristol Medical School, University of Bristol, Bristol, UK

${ }^{5}$ Neonatal Intensive Care Unit, Southmead Hospital, North Bristol NHS Trust, Westbury on Trym, UK

${ }^{6}$ Faculty of Psychology, University of Warsaw, Warszawa, Poland

${ }^{7}$ Ophthalmology, Bristol Eye Hospital, University Hospitals Bristol NHS Foundation Trust, Bristol, UK

${ }^{8}$ Bristol Medical School, Faculty of Health Sciences, University of Bristol, Bristol, UK ${ }^{9}$ Child Psychology, Community Children's Health Partnership, Bristol, UK ${ }^{10}$ Department of Neurosurgery, Great Ormond Street Hospital for Children NHS Foundation Trust, London, UK

${ }^{11}$ Paediatric Neurosurgery, University Hospitals Bristol NHS Foundation Trust, Bristol, UK
}

Twitter Karen Luyt @KarenLuyt and Andrew G Whitelaw @not on twitter

Acknowledgements We thank the families and the children for their support and commitment to the DRIFT trial and for giving their time so generously to advance the knowledge and management of PHVD. This study would not have been possible without their significant contribution. Thank you to the independent trial steering committee for their expert guidance. Agnieszka Rojczyk assisted with cognitive assessments in Poland. Penny Warnes performed the visual assessments on the children in Bristol. The Bristol Randomised Trials Collaboration (BRTC) provided expertise at every stage of the study. CRICBristol provided the facilities for assessment of the children in Bristol.

Contributors Study concept and design: KL, AW, DO, SJ, CW, PB, MM, GK, HEM, WH, KA, IP, SW-C. Acquisition, analysis or interpretation of data: KL, SJ, HEM, GK,
CW, CLL, MM, DO, GJY, PB, WH, KA, IP, AW, AS-C. Drafting of the manuscript: KL, GJY, AW. Critical revision of the manuscript for important intellectual content: $K L$, SJ, CLL, GJY, DO, HEM, GK, CW, PB, WH, MM, SW-C, KA, IP, AS-C, AW. Statistical analysis: GJY, PB. Obtained funding: KL, AW, DO, PB, SJ, CW, GK, MM, SW-C, KA, IP. Administrative, technical or material support: KL, AW, SJ, CLL, KA, IP, SW-C. KL and GJY had full access to all the data in the study and take responsibility for the integrity of the data and the accuracy of the data analysis.

Disclaimer The views expressed are those of the authors and not necessarily those of the NHS, the NIHR or the Department of Health. The funding sources had no role in the design and conduct of the study; collection, management, analysis and interpretation of the data; preparation, review or approval of the manuscript; and decision to submit the manuscript for publication.

Competing interests None declared.

\section{Patient consent for publication Not required.}

Ethics approval Ethical approval was granted by the NHS Health Research Authority, NRES Committee South West - Central Bristol (14/SW/1078) and by the Faculty of Psychology Research Ethics Committee (University of Warsaw). Parents gave written informed consent. The children gave their assent for follow-up.

Provenance and peer review Not commissioned; externally peer reviewed.

Data availability statement Data are available upon reasonable request. Individual participant data that underlie the results reported in this article will be available, after deidentification (text, tables, figures and appendices), to investigators whose proposed use of the data has been approved by an independent review committee ('learned intermediary') identified for this purpose. The data may be used for individual participant data meta-analysis. Proposals should be directed to Karen.Luyt@bristol.ac.uk. Proposals may be submitted up to 5 years following article publication. To gain access, data requestors will need to sign a data access agreement.

Open access This is an open access article distributed in accordance with the Creative Commons Attribution 4.0 Unported (CC BY 4.0) license, which permits others to copy, redistribute, remix, transform and build upon this work for any purpose, provided the original work is properly cited, a link to the licence is given, and indication of whether changes were made. See: https://creativecommons.org/ licenses/by/4.01.

\section{ORCID iDs}

Karen Luyt http://orcid.org/0000-0002-9806-1092

David E Odd http://orcid.org/0000-0002-6416-4966

\section{REFERENCES}

1 Gale C, Statnikov Y, Jawad S, et al. Neonatal brain injuries in England: population-based incidence derived from routinely recorded clinical data held in the National neonatal research database. Arch Dis Child Fetal Neonatal Ed 2018;103:fetalneonatal-2017-313707.

2 van de Bor M, den Ouden L. School performance in adolescents with and without periventricular-intraventricular hemorrhage in the neonatal period. Semin Perinatol 2004;28:295-303.

3 Larroche JC. Post-Haemorrhagic hydrocephalus in infancy. anatomical study. Biol Neonate 1972;20:287-99

4 Murphy BP, Inder TE, Rooks V, et al. Posthaemorrhagic ventricular dilatation in the premature infant: natural history and predictors of outcome. Arch Dis Child Fetal Neonatal Ed 2002:87:37F-41.

5 Whitelaw A, Aquilina K. Management of posthaemorrhagic ventricular dilatation. Arch Dis Child Fetal Neonatal Ed 2012:97:F229-33.

6 Adams-Chapman I, Hansen NI, Stoll BJ, et al. Neurodevelopmental outcome of extremely low birth weight infants with posthemorrhagic hydrocephalus requiring shunt insertion. Pediatrics 2008;121:e1167-77.

7 Brouwer AJ, Brouwer MJ, Groenendaal F, et al. European perspective on the diagnosis and treatment of posthaemorrhagic ventricular dilatation. Arch Dis Child Fetal Neonatal Ed 2012;97:F50-5

8 Limbrick DD, Mathur A, Johnston JM, et al. Neurosurgical treatment of progressive posthemorrhagic ventricular dilation in preterm infants: a 10-year single-institution study. J Neurosurg Pediatr 2010;6:224-30.

9 Mazzola CA, Choudhri AF, Auguste Kl, et al. Pediatric hydrocephalus: systematic literature review and evidence-based guidelines. Part 2: management of posthemorrhagic hydrocephalus in premature infants. J Neurosurg Pediatr 2014;14(Suppl 1):8-23.

10 Whitelaw A, Evans D, Carter M, et al. Randomized clinical trial of prevention of hydrocephalus after intraventricular hemorrhage in preterm infants: brain-washing versus tapping fluid. Pediatrics 2007;119:e1071-8.

11 Whitelaw A, Jary S, Kmita G, et al. Randomized trial of drainage, irrigation and fibrinolytic therapy for premature infants with posthemorrhagic ventricular dilatation: developmental outcome at 2 years. Pediatrics 2010;125:e852-8. 
12 Whitelaw A, Pople I, Cherian S, et al. Phase 1 trial of prevention of hydrocephalus after intraventricular hemorrhage in newborn infants by drainage, irrigation, and fibrinolytic therapy. Pediatrics 2003;111:759-65.

13 Luyt K, Jary S, Lea C, et al. Ten-year follow-up of a randomised trial of drainage, irrigation and fibrinolytic therapy (DRIFT) in infants with post-haemorrhagic ventricular dilatation. Health Technol Assess 2019;23:1-116.

14 Elliot C, Smith P. British ability scales. 3rd edn. London: GL Assessment, 2012.

15 Bayley N. Bayley scales of infant development. 3rd edn. San Antonio, Texas: Harcourt Assessment, 2006.

16 Jary S, Kmita G, Whitelaw A. Differentiating developmental outcome between infants with severe disability in research studies: the role of Bayley developmental quotients. J Pediatr 2011;159:211-4.

17 Houliston MJ, Taguri AH, Dutton GN, et al. Evidence of cognitive visual problems in children with hydrocephalus: a structured clinical history-taking strategy. Dev Med Child Neurol 1999;41:298-306.
18 Henderson S, Sugden D, Barnett A. Movement assessment battery for children (examiners manual). 2nd edn. London: Pearson Assessment, 2007.

19 Palisano R, Rosenbaum P, Walter S, et al. Development and reliability of a system to classify gross motor function in children with cerebral palsy. Dev Med Child Neurol 1997;39:214-23.

20 Goodman R. The strengths and difficulties questionnaire: a research note. J Child Psychol Psychiatry 1997;38:581-6.

21 de Vries LS, Groenendaal F, Liem KD, et al. Treatment thresholds for intervention in posthaemorrhagic ventricular dilation: a randomised controlled trial. Arch Dis Child Fetal Neonatal Ed 2019;104:F70-5.

22 de Vries LS, Liem D, Haastert van I. Randomized trial of early versus late intervention for premature infants with posthemorrhagic ventricular dilatation: developmental outcome at 2 years. Pediatric Academic Societies Meeting, Baltimore, 2019.

23 Schulz M, Bührer C, Pohl-Schickinger A, et al. Neuroendoscopic lavage for the treatment of intraventricular hemorrhage and hydrocephalus in neonates. J Neurosurg Pediatr 2014;13:626-35. 